\title{
Renal Replacement Therapy in a Caribbean Population: The Impact of Biological, Sociocultural and Demographic Factors
}

\author{
Sharlene Sanchez ${ }^{*}$, Aruna Rampersad ${ }^{1}$, Cathy-Lee Jagdeo1, Patrice Ayuen ${ }^{2}$, \\ Chanelle Gunness ${ }^{3}$, George Legall ${ }^{4}$
}

\author{
${ }^{1}$ Department of Medicine, Eric Williams Medical Sciences Complex, Mt Hope, Trinidad \\ ${ }^{2}$ Department of Respiratory Medicine, Nottingham City Hospital, Nottingham, UK \\ ${ }^{3}$ Department of Medicine, San Fernando General Hospital, San Fernando, Trinidad \\ ${ }^{4}$ Faculty of Science and Technology, The University of the West Indies, St Augustine, Trinidad \\ Email: *sanchezsharlene32@yahoo.com
}

\begin{abstract}
How to cite this paper: Sanchez, S., Rampersad, A., Jagdeo, C.-L., Ayuen, P., Gunness, C. and Legall, G. (2018) Renal Replacement Therapy in a Caribbean Population: The Impact of Biological, Sociocultural and Demographic Factors. International Journal of Clinical Medicine, 9, 142-161. https://doi.org/10.4236/ijcm.2018.93013
\end{abstract}

Received: January 22, 2018

Accepted: March 9, 2018

Published: March 12, 2018

Copyright $\odot 2018$ by authors and Scientific Research Publishing Inc. This work is licensed under the Creative Commons Attribution International License (CC BY 4.0).

http://creativecommons.org/licenses/by/4.0/

c) (i) Open Access

\begin{abstract}
Background: The options for renal replacement therapy for end stage renal disease include haemodialysis (HD), peritoneal dialysis (PD) and renal transplantation. In this study demographic, sociocultural and biological factors were assessed over a 1-year period for patients on renal replacement therapy. Methods: This cross-sectional study included all patients 18 years and older and on renal replacement therapy for at least 3 months in Trinidad and Tobago. Five hundred and thirty participants were recruited from our organ transplantation unit, all centres facilitating $\mathrm{PD}$ and a stratified random sample of all HD centres (100 T, 80 PD, 350 HD from October 2015 to October 2016. A questionnaire was administered and included demographics, knowledge and understanding and biological factors impacting on renal replacement therapy. Results: Thirty eight percent of all patients were between 56 to 65 years of age. The Indo Trinidadian population accounted for $51 \%$ of the subjects. $52.5 \%$ were male and $47.5 \%$ were female. From the data, $72 \%$ of patients were diabetic and/or hypertensive. In the transplant recipients, $39 \%$ were diabetic and/or hypertensive and $27 \%$ reported chronic glomerulonephritis as the aetiology of their kidney failure. The diagnosis of chronic kidney disease was made when patients were at end stage renal disease requiring intervention in $84.2 \%$ of persons. The employed population of patients constituted $65 \%$ of renal transplant recipients, $43.75 \%$ of peritoneal dialysis patients and $22.86 \%$ of haemodialysis patients. The patient's physician had the greatest influence on renal replacement therapy choice ( $85.4 \%$ haemodialysis, $85 \%$ peritoneal dialysis, $71 \%$ transplant). Conclusions: In this cohort of 530 patients on renal
\end{abstract}


replacement therapy, knowledge of therapy and physician's influence were some of the factors that impacted on choice of therapy. Most patients were already de novo end stage renal disease when the diagnosis of kidney disease was made. Lifestyle diseases-type 2 diabetes mellitus and hypertension were the main causative diseases contributing to renal failure. This study which is the first in the Caribbean to evaluate biological, sociocultural and demographic factors in patients receiving renal replacement will better assist in prevention strategies and resource allocation.

\section{Keywords}

Renal Replacement Therapy, Sociocultural, Biological

\section{Introduction}

Renal replacement therapy for end stage renal disease includes haemodialysis, peritoneal dialysis and renal transplantation. Many biological, sociocultural, economic and demographic factors affect choice and type of renal replacement therapy in different populations. The choice of modality of renal replacement therapy in Trinidad and Tobago and demographics has not been assessed before. Similar studies have been done in other populations but none in the Caribbean [1]-[16].

A cross sectional study screening high risk patients attending primary care facilities in East Trinidad with undiagnosed chronic kidney disease showed that $53.7 \%$ were classified as having stages $1-3$ chronic kidney disease [7]. A further $18.0 \%$ of asymptomatic participants were found to have stage 3 chronic kidney disease. This highlights the need for sensitization of the public to the detrimental effects of chronic kidney disease, preventative measures and managing risk factors for chronic diseases. This study will also highlight when the diagnosis of chronic kidney disease is made among patients on renal replacement. Each therapy is associated with complications that affect survival and quality of life among patients. Therefore, demographic factors, medical history and complications will be investigated.

In 2006, the Caribbean Renal Registry reported on patients receiving renal replacement therapy in Trinidad and Tobago. Patients diagnosed with end stage renal disease had a male to female ratio of 1.3:1 ranging from 8 - 78 years (mean age 52.5 years). The three commonest causes of renal failure were diabetes mellitus $(28.9 \%)$, primary hypertension (25.3\%), autosomal dominant polycystic kidney disease (3.9\%) and chronic glomerulonephritis (3.9\%) [17]. At that time, the registry did not report renal transplants for Trinidad. There were a total of 436 patients on dialysis, 363 haemodialysis patients and 73 peritoneal dialysis patients. This study will show if the demands for renal replacement therapy has increased over the years and the main modality used.

The renal transplantation journey in Trinidad and Tobago began in 1988 
through the private sector [18]. In 2000, the Human Tissue Transplant Act was passed. With this, came the establishment of the National Organ Transplant Unit in 2006 where donors and recipients could be assessed and managed. From the inception of this unit in January 2006 to December 2015, there have been a total of 148 renal transplantations [18]. A retrospective study from January 2006 to June 2011 showed that one-year, two-year and three-year patient survival rates were $91.46 \%$ (SE 0.04), 89.51\% (SE 0.04) and 86.31\% (SE 0.05), respectively in keeping with acceptable standards [19].

Patients deciding on renal replacement therapy will be empowered to make better decisions with respect to modality of therapy that will inevitably contribute to an improved quality of life.

\section{Methods}

\subsection{Study Description}

This is a cross sectional study on patients receiving renal replacement therapy in Trinidad and Tobago. Patients diagnosed with end stage renal disease can be placed on different treatment modalities. These include: haemodialysis, peritoneal dialysis and renal transplantation. Trinidad and Tobago have all three forms of renal replacement therapy.

\subsection{Study Population}

1) Inclusion criteria

The study population included all patients on renal replacement therapy for at least 3 months and patients 18 years and older. A minimum interval of 3 months is necessary for patient recovery and return to daily life activities after initiation of renal replacement therapy [6] [10] [11].

The diagnosis of end stage renal disease was made with the National Kidney Foundation practice guidelines [20] using an estimated glomerular filtration rate of $\leq 15 \mathrm{mls} / \mathrm{min} / 1.73 \mathrm{~m}^{2}$.

2) Exclusion criteria

Patients excluded were persons less than 18 years and persons receiving renal replacement therapy for $<3$ months.

\subsection{Sample Size Estimation}

The sample size was obtained using a 95\% confidence interval, a margin of error of 0.0025 and a percentage estimation of 0.5 . This gives a minimal sample size of 384 patients on renal replacement therapy: 26 peritoneal dialysis patients, 323 haemodialysis patients (262 private and 61 public) and 35 renal transplant recipients.

\subsection{Recruitment of Subjects}

Haemodialysis and peritoneal dialysis are undertaken at our public institutions. Public hospitals alone manage all peritoneal dialysis patients. Private facilities 
also offer haemodialysis and most patients are funded by the government through the external patient programme. Renal transplantation is accessed through our National Organ Transplant Unit, in the public sector at no cost to the patient.

The subjects were recruited from both public and private institutions from October 2015 to October 2016. Peritoneal dialysis patients were recruited from our public hospitals where this type of dialysis is done. Haemodialysis patients were recruited from private and public facilities across Trinidad. The public facilities included San Fernando General Hospital, Eric Williams Medical Sciences Complex, Port of Spain General Hospital, Scarborough Hospital and Sangre Grande General Hospital. The renal transplant patients were recruited from the National Organ Transplant Unit at the Eric Williams Medical Sciences Complex. The mode of renal replacement therapy for each patient in our population is physician and patient dependent with the nephrologist deciding on the best mode of therapy. Some of the factors affecting choice of therapy in our setting include: financial circumstances, adherence to therapy, age and complications of therapy.

The renal transplantation population consists of a total of 108 successful living kidney transplant recipients from the inception of the National Organ Transplant unit in January 2006 to December 2015. Of this renal transplant population, 100 patients met the inclusion criteria and were included in the study. There are about 90 patients on peritoneal dialysis across Trinidad and Tobago, 80 patients met inclusion criteria and were subjects in this study.

The haemodialysis population presently is approximately over 1000 patients. Among the haemodialysis patients, stratified random sampling was done among private facilities. In October 2015 there were a total of 16 private haemodialysis centres that participated in the government's extended patient programme. These private facilities for sampling purposes were divided into geographical locations using their distribution within each regional health facility of Trinidad and Tobago. These included the North Central Regional Health Facility, North West Regional Health Facility and South West Regional Health Facility. The ratio of distribution was 1:1:2 respectively. Private facilities were therefore sampled by stratified random sampling using these ratios. The haemodialysis patients within the facilities were obtained by convenience sampling. A total of 13 centres were sampled in this study which included all the public centres and 8 private centres.

\subsection{Data Collection Tool}

A demographics questionnaire was employed which gathered information on age, ethnicity, and length of time on renal replacement therapy, employment, income, social support, medical history, substance use, weight, height and previous hospitalizations. Employment, body mass index and medical conditions were classified using the International Standard Classification of Occupations of 
the United Nations [21], the World Health Organization Global Database on Body Mass Index [22] and the International Statistical Classification of Diseases and Related Health Problems $10^{\text {th }}$ Revision [23] respectively. Body mass index for obese patients was further categorized for persons of Asian $\left(25 \mathrm{~kg} / \mathrm{m}^{2}\right)$ and Afro-Caribbean descents $\left(27 \mathrm{~kg} / \mathrm{m}^{2}\right)$ [24].

\subsection{Ethics and Consent}

Ethical approvals and permissions were obtained for all sampled facilities. Approvals from the ethics committees from the respective regional health facilities were obtained. The University of the West Indies, Research Ethics Committee also granted their approval for this project.

Data collection commenced October 2015 and was completed in October 2016. Strict confidentiality of information was maintained throughout this study. The purpose of the study and content of questionnaires was explained fully to patients before the interview. Informed consent from all participants in this study was obtained. Questionnaires were administered in an interview-based format to patients.

\subsection{Statistical Analysis}

Statistical Package for the Social Sciences (SPSS) version 24 was used for statistical analysis. Inter-collector reliability was tested periodically.

\section{Results}

\subsection{Demographics}

There were a total of 530 patients on renal replacement therapy in this study between October 2015 and October 2016. Of these, 350 were on haemodialysis, 80 were on peritoneal dialysis and 100 were kidney transplant recipients. Thirty eight percent of all patients were between 56 to 65 years of age (Table 1). 52.5\% were male and $47.5 \%$ were female (Table 2 ). The Indo Trinidadian population accounted for $51 \%$ of the subjects (Figure 1).

\subsection{Biologic}

Sixty four percent of patients were found to be obese (Table 3). In a study by Tillin et al, that followed over 2500 persons, body mass index cut off levels of 25 in persons of Asian descent and 27 in those of African Caribbean descents posed equivalent risks to those of European descent with a cut off value of 30 [24]. These values were used in this study for persons of these descents.

The diagnosis of chronic kidney disease was made when patients were already at end stage renal disease requiring intervention in $84.2 \%$ of persons. Primary hypertension and/or diabetes mellitus were reported as the reason for chronic kidney disease in $72 \%$ of the population on renal replacement therapy.

Sixty seven percent of haemodialysis patients had permanent catheters as their primary access site for dialysis. Among these patients, 51\% were told they were 
hospitalized for investigation and treatment of catheter related blood stream infections.

Table 1. Age of patients receiving renal replacement therapy.

All modalities Haemodialysis Peritoneal Dialysis Renal Transplant
Characteristic Percent (\%) patients Percent (\%) patients Percent (\%) patients Percent (\%) patients
$\mathrm{N}=530$
$\mathrm{H}=350$
$\mathrm{P}=80$
$\mathrm{T}=100$

\begin{tabular}{ccccc}
\hline Age & & & & \\
18 to 25 years & 5.70 & 3.70 & 7.50 & 11.00 \\
26 to 35 years & 11.90 & 10.80 & 7.50 & 19.00 \\
36 to 45 years & 12.10 & 9.10 & 15.00 & 20.00 \\
46 to 55 years & 20.60 & 18.60 & 18.80 & 29.00 \\
56 to 65 years & 37.50 & 45.70 & 31.30 & 14.00 \\
66 to 75 years & 11.30 & 11.50 & 16.20 & 7.00 \\
$>76$ years & 0.90 & 0.60 & 3.70 & 0.00
\end{tabular}

HD-haemodialysis, PD-peritoneal dialysis, T-renal transplantation.

Table 2. Gender Distribution of Patients Receiving Renal Replacement Therapy.

\begin{tabular}{|c|c|c|c|c|}
\hline & All modalities & Haemodialysis & Peritoneal Dialysis & Renal Transplant \\
\hline Characteristic & Percent (\%) patients & Percent (\%) patients & Percent (\%) patients & Percent (\%) patients \\
\hline & $\mathrm{N}=530$ & $\mathrm{H}=350$ & $\mathrm{P}=80$ & $\mathrm{~T}=100$ \\
\hline \multicolumn{5}{|l|}{ Sex } \\
\hline Male & 52.50 & 52.30 & 46.30 & 58.00 \\
\hline Female & 47.50 & 47.70 & 53.70 & 42.00 \\
\hline
\end{tabular}

HD-haemodialysis, PD-peritoneal dialysis, T-renal transplantation.

\section{Percent}

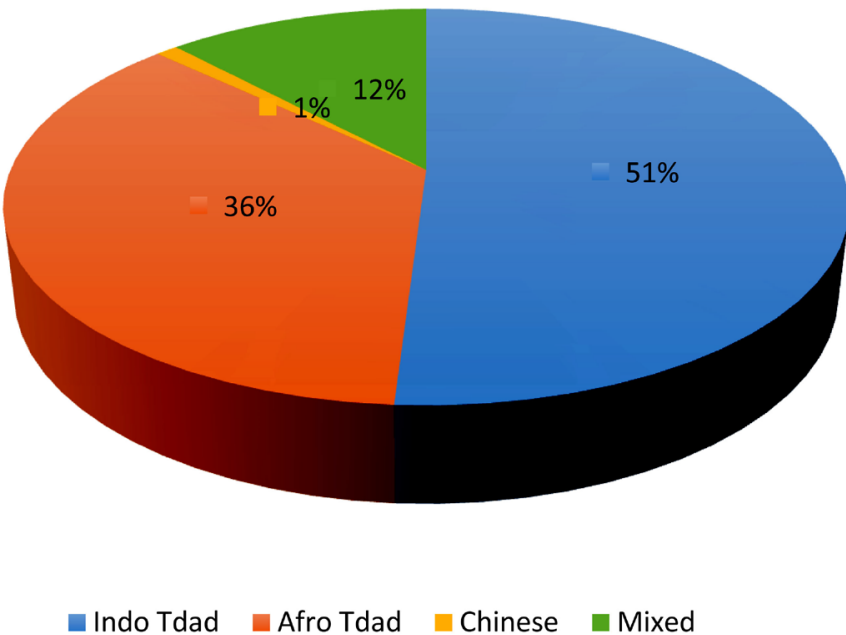

Figure 1. Ethnicity of Patients on Renal Replacement Therapy Indo Tdad: Indo Trinidadian, Afro Tdad: Afro Trinidadian. 
Table 3. Body mass index of patients receiving renal replacement therapy.

\begin{tabular}{ccccc} 
Characteristic & $\begin{array}{c}\text { All modalities percent (\%) } \\
\text { patients } \mathrm{N}=530\end{array}$ & $\begin{array}{c}\text { Haemodialysis percent (\%) } \\
\text { patients } \mathrm{H}=350\end{array}$ & $\begin{array}{c}\text { Peritoneal dialysis percent } \\
\text { (\%) patients } \mathrm{P}=80\end{array}$ & $\begin{array}{c}\text { Renal transplant percent } \\
(\%) \text { patients } \mathrm{T}=100\end{array}$ \\
\hline Body mass index & & & & \\
Underweight $(<18.50)$ & 4.500 & 26.94 & 42.50 & 6.00 \\
Normal $(18.50-24.99)$ & 28.90 & 22.10 & 71.30 & 41.00 \\
Overweight $(\geq 25.00)$ & 2.74 & 12.74 & 39.30 & 48.00 \\
Obese $(\geq 30)$ & 63.86 & 38.30 & & \\
Asian descent $(\geq 25.00)^{1}$ & & & & \\
Afro-Caribbean descent $(\geq 27.00)^{1}$ & & &
\end{tabular}

HD-haemodialysis, PD-peritoneal dialysis, T-renal transplantation. ${ }^{1}$ T.Tillin, N. Sattar, I.F Godsland, A.D Hughes, N. Chaturvedi et al. Ethnicity-specific obesity cut-points in the development of type 2 diabetes-a prospective study including three ethnic groups in the United Kingdom. Diabet Med. 2015; 32: 226-234.

Table 4. Patients previously on other forms of renal replacement therapy before current therapy.

\begin{tabular}{|c|c|c|c|c|}
\hline Factor & $\begin{array}{l}\text { All modalities \% } \\
\text { patients } \\
\mathrm{N}=530\end{array}$ & $\begin{array}{c}\text { Haemodialysis \% } \\
\text { patients } \\
\text { HD }=350\end{array}$ & $\begin{array}{l}\text { Peritoneal dialysis \% } \\
\text { patients } \\
\text { PD }=80\end{array}$ & $\begin{array}{c}\text { Transplant } \% \\
\text { patients } \\
\mathrm{T}=100\end{array}$ \\
\hline \multicolumn{5}{|l|}{ Prior form of renal replacement therapy (not in an acute setting) } \\
\hline Not on any form of renal replacement therapy before & 81.90 & 98.57 & 78.75 & 20.00 \\
\hline Haemodialysis & 16.22 & N/A & 17.50 & 76.00 \\
\hline Peritoneal dialysis & 0.56 & 0.00 & N/A & 4.00 \\
\hline Renal transplant & 1.32 & 1.43 & 3.75 & N/A \\
\hline
\end{tabular}

HD-haemodialysis, PD-peritoneal dialysis, T-renal transplantation; N/A-not applicable.

Seven percent of transplant recipients reported a diagnosis of post-transplant erythrocytosis. Eleven reported a diagnosis of new onset Diabetes Mellitus after transplant. Nineteen percent of transplant patients reported distressing symptoms when responding to the kidney transplant questionnaire. The most distressing symptoms were those related to infections. Other symptoms reported included dyspnoea, headaches and altered bowel habit.

Thirty four percent of the renal transplant recipients had been transplanted for more than 5 years compared to $8 \%$ of haemodialysis patients and $12.5 \%$ of peritoneal dialysis patients who were on their respective forms of renal replacement for more than 5 years (Table 4).

After starting renal replacement therapy, $30.5 \%$ of haemodialysis patients had 2 or more hospitalizations, $7.5 \%$ of peritoneal dialysis patients and $6 \%$ of renal transplant recipients had 2 or more hospitalizations post renal replacement therapy. The common reasons for hospitalizations reported among haemodialysis patients were catheter related blood stream infections (41.4\%) and fluid overload secondary to chronic renal failure (37.7\%).

Sixty seven percent of haemodialysis patients were using more than 10 medications compared to a smaller proportion of peritoneal dialysis and transplant recipients, $7.5 \%$ and $6 \%$ respectively (Table 5 ). Alcohol consumption and cigarette use are shown in Table 6 and Table 7. 
Table 5. Medications used by patients receiving renal replacement therapy.

\begin{tabular}{|c|c|c|c|c|}
\hline Factor & $\begin{array}{l}\text { All modalities \% patients } \\
\qquad \mathrm{N}=530\end{array}$ & $\begin{array}{l}\text { Haemodialysis } \% \text { patients } \\
\qquad \mathrm{HD}=350\end{array}$ & $\begin{array}{l}\text { Peritoneal dialysis } \% \text { patients } \\
\text { PD }=80\end{array}$ & $\begin{array}{c}\text { Transplant } \% \text { patients } \\
\mathrm{T}=100\end{array}$ \\
\hline \multicolumn{5}{|c|}{ Medications } \\
\hline 1 to 3 & 3.58 & 0.29 & 11.25 & 9.00 \\
\hline 4 to 6 & 18.69 & 6.85 & 37.50 & 45.00 \\
\hline 7 to 10 & 31.32 & 26.00 & 43.75 & 40.00 \\
\hline$>10$ & 46.41 & 66.86 & 7.50 & 6.00 \\
\hline
\end{tabular}

HD-haemodialysis, PD-peritoneal dialysis, T-renal transplantation.

Table 6. Number of years of alcohol consumption by patients.

\begin{tabular}{|c|c|c|c|c|}
\hline Factor & $\begin{array}{l}\text { All modalities \% patients } \\
\qquad \mathrm{N}=530\end{array}$ & $\begin{array}{l}\text { Haemodialysis \% patients } \\
\text { HD }=350\end{array}$ & $\begin{array}{l}\text { Peritoneal dialysis } \% \text { patients } \\
\text { PD }=80\end{array}$ & $\begin{array}{c}\text { Transplant } \% \text { patients } \\
\mathrm{T}=100\end{array}$ \\
\hline \multicolumn{5}{|c|}{ Number of years consuming alcohol } \\
\hline No alcohol consumed & 78.87 & 76.85 & 77.50 & 87.00 \\
\hline$\leq 5$ years & 2.64 & 1.71 & 7.50 & 2.00 \\
\hline$>5$ years, $\leq 10$ years & 6.04 & 6.80 & 7.50 & 2.00 \\
\hline$>10$ years, $\leq 15$ years & 2.45 & 2.57 & 3.75 & 7.00 \\
\hline$>15$ years, $\leq 20$ years & 7.55 & 8.86 & 2.50 & 1.00 \\
\hline$>20$ years & 2.45 & 3.14 & 1.25 & 1.00 \\
\hline
\end{tabular}

HD-haemodialysis, PD-peritoneal dialysis, T-renal transplantation.

Table 7. Number of pack years of cigarette use.

\begin{tabular}{|c|c|c|c|c|}
\hline Factor & $\begin{array}{l}\text { All modalities \% patients } \\
\qquad \mathrm{N}=530\end{array}$ & $\begin{array}{c}\text { Haemodialysis \% patients } \\
\text { HD }=350\end{array}$ & $\begin{array}{l}\text { Peritoneal dialysis \% patients } \\
\qquad \mathrm{PD}=80\end{array}$ & $\begin{array}{c}\text { Transplant \% patients } \\
\mathrm{T}=100\end{array}$ \\
\hline \multicolumn{5}{|l|}{ Cigarette smoking } \\
\hline Never smoked & 89.10 & 84.86 & 95.00 & 99.00 \\
\hline$<10$ pack years & 0.75 & 0.85 & 0.00 & 1.00 \\
\hline$<20$ pack years, $\geq 10$ pack years & 3.20 & 4.00 & 3.75 & 0.00 \\
\hline$<30$ pack years, $\geq 20$ pack years & 3.02 & 4.29 & 1.25 & 0.00 \\
\hline$<40$ pack years, $\geq 30$ pack years & 0.57 & 0.85 & 0.00 & 0.00 \\
\hline$\geq 40$ pack years & 3.40 & 5.14 & 0.00 & 0.00 \\
\hline
\end{tabular}

HD-haemodialysis, PD-peritoneal dialysis, T-renal transplantation.

\subsection{Sociocultural}

In the entire population of patients, $17.7 \%$ were single and $54.7 \%$ were married (Table 8 ). $51.1 \%$ of patients were Christian, $33.8 \%$ were Hindu and $11.5 \%$ were Muslim (Table 9).

\subsection{Employment and Education}

A greater proportion of transplant recipients completed or was completing tertiary level education (Figure 2). After receiving renal replacement therapy for end stage renal disease, $21.7 \%$ of haemodialysis patients in the 18 to 65 years age 
Table 8. Marital status of patients receiving renal replacement therapy.

\begin{tabular}{ccccc}
\hline Characteristic & $\begin{array}{c}\text { All modalities percent (\%) } \\
\text { patients } \mathrm{N}=530\end{array}$ & $\begin{array}{c}\text { Haemodialysis percent (\%) } \\
\text { patients } \mathrm{H}=350\end{array}$ & $\begin{array}{c}\text { Peritoneal dialysis percent (\%) } \\
\text { patients } \mathrm{P}=80\end{array}$ & $\begin{array}{c}\text { Renal transplant percent } \\
\text { patients } \mathrm{T}=100\end{array}$ \\
\hline Marital status & & & & \\
Single & 17.74 & 13.43 & 16.25 & 34.00 \\
Married & 54.72 & 52.28 & 60.00 & 1.25 \\
Divorced & 2.83 & 3.43 & 16.25 & 59.00 \\
Common law & 22.83 & 29.70 & 6.25 & 4.00 \\
Widowed & 1.89 & 1.14 & & 1.00 \\
\hline
\end{tabular}

HD-haemodialysis, PD-peritoneal dialysis, T-renal transplantation.

Table 9. Religious beliefs of patients receiving renal replacement therapy.

\begin{tabular}{ccccc}
\hline Characteristic & $\begin{array}{c}\text { All modalities percent (\%) } \\
\text { patients } \mathrm{N}=530\end{array}$ & $\begin{array}{c}\text { Haemodialysis percent (\%) } \\
\text { patients } \mathrm{H}=350\end{array}$ & $\begin{array}{c}\text { Peritoneal dialysis percent (\%) } \\
\text { patients } \mathrm{P}=80\end{array}$ & $\begin{array}{c}\text { Renal transplant percent (\%) } \\
\text { patients } \mathrm{T}=100\end{array}$ \\
\hline Religion & & & & \\
Christian & 51.13 & 54.75 & 60.00 & 62.00 \\
Hindu & 33.77 & 35.14 & 31.25 & 31.00 \\
Muslim & 11.51 & 6.28 & 3.75 & 6.00 \\
Other & 2.83 & 3.42 & 2.50 & 1.00 \\
No religion & 0.75 & 0.57 & 2.50 & 0.00 \\
\hline
\end{tabular}

HD-haemodialysis, PD-peritoneal dialysis, T-renal transplantation.

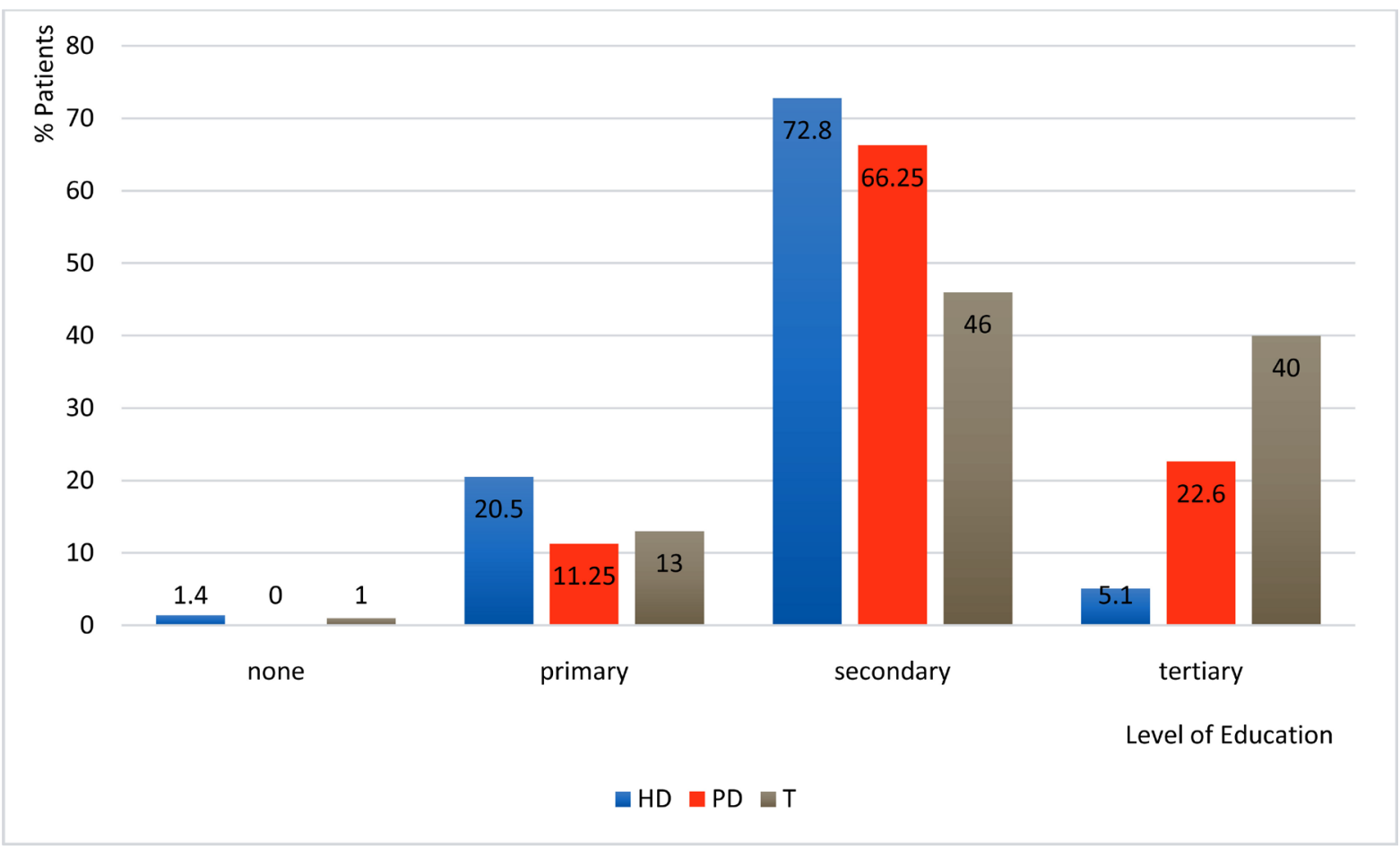

HD-haemodialysis, PD-peritoneal dialysis, T-renal transplantation.

Figure 2. Level of education completed or completing and mode of renal replacement therapy. 
group reported that they were able to return to work or obtain employment because of starting renal replacement therapy. Among the same age group, 36.25\% of the peritoneal dialysis patients and $63 \%$ of the renal transplant recipients were able to return to work or obtain employment. $65 \%$ of renal transplant recipients were employed (Table 10). The renal transplant recipients had a greater proportion of positions as managers or professionals (Table 11).

\subsection{Economic}

Among the haemodialysis patients $20 \%$ were either current or prior recipients of social welfare, $20 \%$ of the peritoneal dialysis patients received or previously received social welfare and $2 \%$ of the transplant recipients were either prior or present recipients of social welfare. Fifty nine percent of haemodialysis patients reported they earned \$1000 TT (Trinidad and Tobago) dollars or less each month, $32.5 \%$ of the peritoneal dialysis patients earned within this bracket while $27 \%$ of transplant patients reported this monthly income (Table 12).

\subsection{Knowledge and Understanding of Modalities}

Figure 3 shows that among all groups of patients, the person having the most

Table 10. Employment status of patients on renal replacement therapy.

\begin{tabular}{ccccc}
\hline Factor & $\begin{array}{c}\text { All modalities percent } \\
(\%) \text { patients } \mathrm{N}=530\end{array}$ & $\begin{array}{c}\text { Haemodialysis percent } \\
(\%) \text { patients } \mathrm{H}=350\end{array}$ & $\begin{array}{c}\text { Peritoneal dialysis percent } \\
(\%) \text { patients } \mathrm{P}=80\end{array}$ & $\begin{array}{c}\text { Renal transplant percent } \\
(\%) \text { patients } \mathrm{T}=100\end{array}$ \\
\hline Employment & & & & 22.50 \\
Not employed or pursuing educational goals & 43.77 & 54.00 & 43.75 & 11.00 \\
Employed & 33.96 & 22.86 & 13.75 & 65.00 \\
Pursuing educational goals & 12.08 & 14.29 & 20.00 & 14.00 \\
Retired & 10.57 & 16.00 & 10.00 \\
\hline
\end{tabular}

HD-haemodialysis, PD-peritoneal dialysis, T-renal transplantation.

Table 11. Occupation of patients on renal replacement therapy.

\begin{tabular}{|c|c|c|c|c|}
\hline Factor & $\begin{array}{l}\text { All modalities } \% \\
\text { patients } \mathrm{N}=530\end{array}$ & $\begin{array}{l}\text { Haemodialysis \% } \\
\text { patients } \mathrm{H}=350\end{array}$ & $\begin{array}{c}\text { Peritoneal dialysis } \% \\
\text { patients } \mathrm{P}=80\end{array}$ & $\begin{array}{c}\text { Transplant } \% \\
\text { patients } \mathrm{T}=100\end{array}$ \\
\hline \multicolumn{5}{|l|}{ Occupation $^{1}$} \\
\hline Managers & 4.15 & 2.57 & 1.25 & 12.00 \\
\hline Professionals & 7.92 & 2.85 & 11.25 & 23.00 \\
\hline Technicians and associate professionals & 1.70 & 0.29 & 1.25 & 7.00 \\
\hline Clerical support workers & 3.40 & 1.43 & 6.25 & 8.00 \\
\hline Service and sale workers & 7.55 & 8.57 & 6.25 & 5.00 \\
\hline Skilled agricultural forestry and fishery workers & 0.38 & 0.28 & 0.00 & 1.00 \\
\hline Craft and related trade worker & 4.91 & 2.00 & 6.25 & 14.00 \\
\hline Plant and machine operators and assemblers & 3.58 & 1.71 & 13.75 & 2.00 \\
\hline Elementary occupations & 4.71 & 5.70 & 0.00 & 5.00 \\
\hline Armed forces occupation & 0.57 & 0.28 & 1.25 & 1.00 \\
\hline
\end{tabular}

HD-haemodialysis, PD-peritoneal dialysis, T-renal transplantation. ${ }^{1}$ International Standard Classification of Occupations of the United Nations. ISCO 08. http://www.ilo.org/public/english/bureau/stat/isco/ 
Table 12. Income of patients receiving renal replacement therapy.

\begin{tabular}{|c|c|c|c|c|}
\hline Characteristic & $\begin{array}{l}\text { All modalities percent } \\
\text { (\%) patients } \mathrm{N}=530\end{array}$ & $\begin{array}{l}\text { Haemodialysis percent } \\
\text { (\%) patients } \mathrm{H}=350\end{array}$ & $\begin{array}{l}\text { Peritoneal dialysis percent } \\
\text { (\%) patients } \mathrm{P}=80\end{array}$ & $\begin{array}{c}\text { Renal transplant percent } \\
\text { (\%) patients } \mathrm{T}=100\end{array}$ \\
\hline \multicolumn{5}{|l|}{ Income } \\
\hline$\leq \$ 1000 \mathrm{TT}$ & 46.98 & 58.51 & 32.50 & 27.00 \\
\hline$>\$ 1000 \mathrm{TT}, \leq \$ 4000 \mathrm{TT}$ & 12.83 & 15.37 & 11.25 & 1.00 \\
\hline$>\$ 4000 \mathrm{TT}, \leq \$ 8000 \mathrm{TT}$ & 30.57 & 24.51 & 37.50 & 42.00 \\
\hline$>\$ 8000 \mathrm{TT}, \leq 12,000 \mathrm{TT}$ & 8.68 & 1.77 & 18.75 & 25.00 \\
\hline$>12,000 \mathrm{TT}$ & 0.94 & 0.00 & 0.00 & 5.00 \\
\hline
\end{tabular}

HD-haemodialysis, PD-peritoneal dialysis, T-renal transplantation.

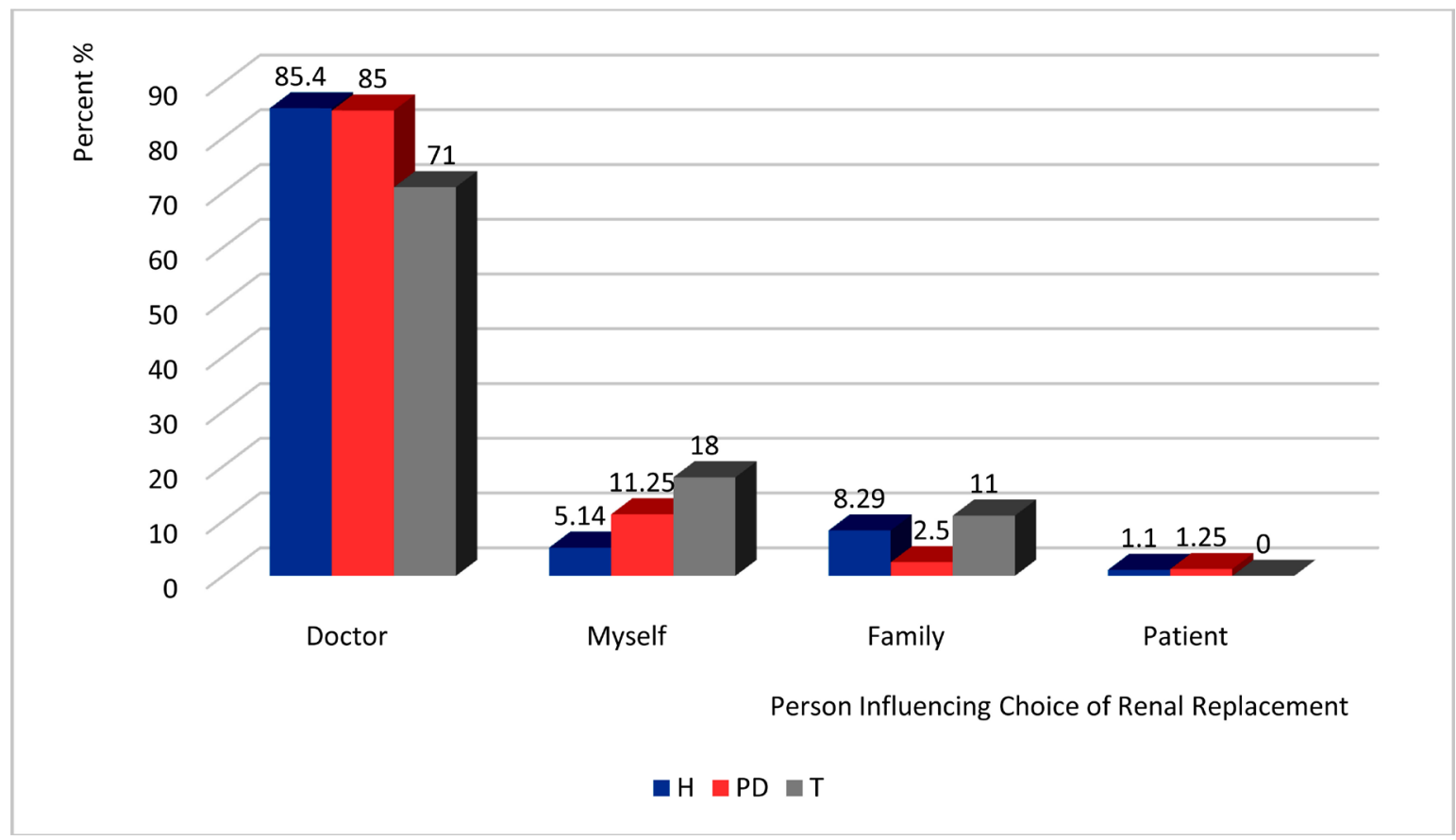

HD-haemodialysis, PD-peritoneal dialysis, T-renal transplantation.

Figure 3. Persons influencing a patient's choice of renal replacement therapy.

influence on a patient when deciding on a modality of renal replacement therapy was the patient's physician (85.4\% haemodialysis, $85 \%$ peritoneal dialysis, $71 \%$ transplant). On assessing patients' knowledge and understanding of their present therapy, $61.5 \%$ of patients said they felt completely knowledgeable about their present therapy (Table 13).

\section{Discussion}

Treatment modalities for end stage renal disease differ around the world. In Norway and Iceland, more than $60 \%$ of patients have been transplanted. Thirty percent of patients in the United States with end stage renal disease have renal transplants and in Asian and Eastern European countries, it is less than $5 \%$. Also, in places like Japan, $91 \%$ of patients use arteriovenous fistulas, dialyze in 
Table 13. Knowledge and understanding of present therapy.

\begin{tabular}{|c|c|c|c|c|}
\hline Factor & $\begin{array}{l}\text { All modalities } \% \\
\text { patients } \mathrm{N}=530\end{array}$ & $\begin{array}{c}\text { Haemodialysis } \% \\
\text { patients } \mathrm{HD}=350\end{array}$ & $\begin{array}{c}\text { Peritoneal } \\
\text { dialysis \% } \\
\text { patients PD = } 80\end{array}$ & $\begin{array}{c}\text { Transplant } \% \\
\text { patients } \mathrm{T}=100\end{array}$ \\
\hline \multicolumn{5}{|l|}{ Pre-renal replacement therapy counselling by doctor (responses) } \\
\hline I felt completely knowledgeable about my present therapy (full) & 61.51 & 50.00 & 80.00 & 92.00 \\
\hline I had a good idea about my present therapy (good) & 30.57 & 32.57 & 13.75 & 6.00 \\
\hline I felt somewhat knowledgeable about my present therapy (somewhat) & 5.85 & 13.71 & 1.25 & 2.00 \\
\hline I felt I knew a little about my present therapy (little) & 1.13 & 2.86 & 2.50 & 0.00 \\
\hline I had no idea about my present therapy (none) & 0.94 & 0.86 & 2.50 & 0.00 \\
\hline
\end{tabular}

HD-haemodialysis, PD-peritoneal dialysis, T-renal transplantation.

the supine position and receive haemodialysis for more than 4 hours to ensure reimbursement [25].

In our population, less than $10 \%$ of patients with end stage renal disease are transplanted. Fifty six percent of patients on haemodialysis have arteriovenous fistulas and our centres usually dialyze patients for a maximum of 3 hours in a semi erect position. There is only one transplant centre in Trinidad that is incapable of facilitating the demands of a growing population with end stage renal disease. Peritoneal dialysis patients need to have acceptable living situations that is under strict assessment before a decision is made for initiating peritoneal dialysis. Seventy three percent of patients receiving transplants were on haemodialysis prior to receiving a renal transplant. In our population haemodialysis is the most accessible form of renal replacement therapy.

\subsection{Comorbidities}

Among all patients in our study, $72 \%$ were diabetic and/or hypertensive. In the transplant recipients, $39 \%$ were diabetic and/or hypertensive and $27 \%$ reported chronic glomerulonephritis as the aetiology of their kidney failure. This study showed that the diagnosis of chronic kidney disease was made when patients were at end stage renal disease requiring intervention in $84.2 \%$ of persons. These numbers may possibly be even higher since some patients diagnosed with end stage renal disease do not wish for any therapy and are managed conservatively.

Again, this emphasizes the need for public awareness and education on controlling the risk factors associated with lifestyle diseases.

\subsection{Medications}

Eighty percent of transplant patients, $32.5 \%$ of haemodialysis patients and $81.25 \%$ of peritoneal dialysis patients were using between 4 and 10 medications. Sixty seven percent of haemodialysis patients were using greater than 10 medications. A greater proportion of patients using 10 medications or less were in the transplant (94\%) and peritoneal dialysis (92.5\%) groups. Patients on haemodialysis may have more comorbidities than patients on other modalities hence the 
need for a greater number of medications. There were $62.5 \%$ of type 2 diabetics among patients on haemodialysis (HD) in this study. Forty eight percent of patients on peritoneal dialysis (PD) were type 2 diabetics and $11 \%$ of renal transplant recipients were type 2 diabetics. The proportion of patients who were hypertensive was greater in the dialysis group (HD: 76.3\%, PD: 73.5\%, T: 39\%). Furthermore, patients on haemodialysis usually require supplementation of calcium, vitamin D, erythropoietin and phosphate binders. This may mean that patients on this form of renal replacement therapy will inevitably be on a greater number of medications than others.

\subsection{Age among Patients on HD}

Sixty six percent of patients with arteriovenous fistulas or grafts were more than 55 years. Seventy seven percent of patients with arteriovenous fistulas or grafts were more than 45 years. One study has shown that younger patients on haemodialysis have better quality of life scores [26]. In this study, $15.5 \%$ of the patients on haemodialysis aged between 18 to 35 years had arteriovenous fistulas or grafts. Patients who were on haemodialysis for a longer time, possibly were able to obtain an arteriovenous fistulas or graft before others because of the length of time waiting in the hospital system.

\subsection{Access Type for HD}

It has been well recognized in many studies that arteriovenous fistulas and arteriovenous grafts are associated with fewer complications and an improved quality of life for patients on long term haemodialysis [27]. In a study of 1563 patient on haemodialysis, patients with arteriovenous fistulas reported greater physical activity and energy, better emotional and social well-being, fewer symptoms, better sleep, less effect of dialysis and burden of kidney disease [28].

\subsection{Body Mass Index and Haemodialysis Patients}

Adipose tissue provides more tumour necrosis factor alpha soluble receptors that attenuate the adverse effects of tumour necrosis factor. Overweight individuals have higher lipoprotein concentrations, which counteract the inflammatory effects of circulating endotoxins [29]. Reductions in total body fat are associated with decreased humoral immunity [30]. Overweight and obese individuals have a higher absolute amount of muscle mass. This increased amount of lean tissue might confer an additional protective edge during times of catabolism which is common in haemodialysis patients [31] [32] [33].

\subsection{Socioeconomic Factors}

\subsubsection{Employment}

The employed population of patients constituted $65 \%$ of renal transplant recipients $43.75 \%$ of peritoneal dialysis patients and $22.86 \%$ of haemodialysis patients. Renal transplant recipients possibly post transplantation because of the de- 
creased demands of this mode of renal replacement were better able to obtain and seek employment. Sixty three percent of renal transplant patients from this study were able to obtain employment or return to their prior occupation after renal transplantation. In the haemodialysis population, $21.7 \%$ obtained employment or returned to their prior occupation and $36.3 \%$ of the peritoneal dialysis patients obtained employment or returned to their prior occupation. Transplantation provided a means of upward mobility and improvement of socioeconomic status compared to other modes of renal replacement therapy which was similar in other studies [5] [9] [10] [14]. In a study done in 2009 that compared employment and modes of renal replacement therapy, unemployment was increased in patients on haemodialysis and peritoneal dialysis but greater in the peritoneal dialysis group while the kidney transplant recipients had a reduction in unemployment [14].

\subsubsection{Income}

Transplant patients were earning in the higher income brackets again suggesting the upward mobility in socioeconomic status with this mode of renal replacement therapy. Other studies have shown a significant relationship between a higher socioeconomic class and a better quality of life [9].

\subsection{Knowledge and Understanding of Renal Replacement Therapy}

Fadem et al showed that external influences such as physician's recommendations affected how patients choose a therapy and disclosed a general preference for physician-based education [34]. In this study, the patient's physician had the greatest influence on renal replacement therapy choice. Patients with a greater knowledge and understanding of their present therapy may feel more empowered to make decisions and directly contribute and initiate other aspects of management like lifestyle modifications, adherence with medications and follow up visits at clinic.

Educating patients on available options of renal replacement plays a pertinent role in assisting patients with managing chronic kidney disease and the comorbidities associated with it. There are studies that have shown a general lack of knowledge and understanding of the options available for renal replacement therapy [35] [36] [37] [38]. It was found that $74.7 \%$ of patients with a good level of knowledge and understanding of renal replacement therapy attained at least a secondary level of education.

\subsection{Selection of Patients for Renal Replacement Therapy}

The selection of patients for each modality of renal replacement therapy may differ because of the specific requirements of each therapy. All modes of renal replacement are available to our population through the public sector.

Renal transplantation is a mode of renal replacement that requires a thorough pre-emptive evaluation of cardiac and vascular status. In our transplant unit, patients are extensively managed and assessed by the transplant surgeon, vascular 
surgeon, nephrologists, transplantation coordinator, dietician, dentist, psychiatrist, social worker and an ethics committee over several months before the transplantation procedure. Adequate optimization of each patient and all comorbidities are stringently managed by expert care. Pre-transplantation counselling is expansive and potential recipients whether on the live or cadaveric waiting list have several lecture sessions with their transplant coordinator and social worker. The pre-transplantation experience differs vastly from persons starting other forms of renal replacement.

When compared to patients on dialysis, haemodialysis is a more feasible option than peritoneal dialysis. There are very few contraindications to haemodialysis when patients are diagnosed with end stage renal disease. It is readily available in the acute setting and is the least time consuming of the three modalities of renal replacement therapy, requiring at least a temporary catheter to initiate therapy. Peritoneal dialysis however needs greater medical evaluation and assesses patients for conditions that may predispose them to developing peritonitis. Peritoneal dialysis also demands a patient to have a certain minimal standard of living to properly perform this therapy in a home setting. As such, patients are routinely evaluated and cleared by their medical social worker prior to commencing peritoneal dialysis. In order to achieve the full therapeutic goals of peritoneal dialysis patients are fully counselled and educated on this form of renal replacement therapy. The recognition of complications and management of comorbidities is highlighted and reinforced by peritoneal dialysis nurses regularly. Patients on peritoneal dialysis and those receiving renal transplants may be more motivated and empowered in managing their kidney disease than haemodialysis patients. Social support networks are needed for all patients on renal replacement but because of the selectivity of each mode, patients on haemodialysis possibly have the least social support.

Of the three modalities of renal replacement therapy, renal transplantation may be the most selective. This highly specific selection of patients on each modality was reflected in this study. There were $62.5 \%$ of type 2 diabetics among patients on haemodialysis (HD), $48 \%$ on peritoneal dialysis (PD) and $11 \%$ receiving renal transplants $(\mathrm{T})$. Hypertension was greater in the dialysis group (HD: $76.3 \%$, PD: 73.5\%, T: 39\%). Thirty four percent of renal transplant recipients had their transplants for $>5$ years compared to $8 \%$ of haemodialysis patients and $12.5 \%$ of peritoneal dialysis patients. Thirty percent of patients aged 18 to 35 years were renal transplant recipients, followed by $15 \%$ of peritoneal dialysis patients and $14.5 \%$ of patients on haemodialysis. This may suggest that younger patients and those with fewer comorbidities are likely to be selected as renal transplant candidates. Our transplant patients were also more likely to be employed and earning in the higher income brackets. This could be because of upward mobility secondary to an improved quality of life after transplantation or potential recipients initially belonging to higher socioeconomic groups than patients on other modes of renal replacement therapy. 
Our transplantation programme in Trinidad and Tobago is government funded and referral based. Donors both living, and deceased must meet specific criteria for eligibility. Worldwide, other transplantation centres may have the added bias of selection of patients based on socioeconomic status since privately, access to transplantations may be greater in persons with insurance or those in the higher income brackets. In a report analysing the data of 600,000 patients listed in the United Network for Organ Sharing database (UNOS) from 2000 to 2013, transplant centres allowed listing of patients at multiple centres. This was initially allowed to facilitate patients from rural areas. However, the practice eventually favoured wealthier patients because they were able to afford registration at multiple sites making them more likely to be transplanted than someone in greater need of transplantation [39].

All transplantation centres should be ethical in their selection of patients for transplantation and should be subjected to regular auditing of transplanted candidates.

Thirty four percent of transplant recipients had allografts for more than 5 years, the remaining $66 \%$ of patients were transplanted for 5 years or less. This may mean that the survival of transplant recipients may be better than patients on other modes of renal replacement. Furthermore, when current time period on therapy was analysed, $19 \%$ of patients with renal transplants had their grafts for greater than 10 years, followed by $2.3 \%$ of patients on haemodialysis and $1.3 \%$ of patients on peritoneal dialysis There are studies that have shown an improvement in morbidity and mortality with renal transplantation over dialysis [40] [41].

Sixty three percent of transplant recipients, $21.71 \%$ of HD patients and $32.5 \%$ of PD patients between the ages of 18 and 65 years were able to return to work or obtain employment after initiating renal replacement therapy. In a prospective study done 20 years ago on cost utility of renal transplantation in Canada, it was found that over two years, transplantation was both less costly and more effective than dialysis. Employment opportunities were the greatest for transplant patients also [10].

Our transplantation programme in Trinidad and Tobago established only 10 years ago is small compared to other transplantation units with a maximum of only 2 live donor transplantations being done monthly and deceased donor renal transplantations taking place on availability and criteria fulfilment. In this study, out of the 100 renal transplant patients meeting the inclusion criteria, 24 were recipients from deceased donors. With larger transplantation programmes, cost per transplantation may be reduced.

\subsection{Limitations and Future Research}

Our dialysis centres assess each patient and regulates each dialysis prescription to achieve good clearances. However, this study did not assess objectively adequacy of haemodialysis for each patient by analysing clearances among haemo- 
dialysis and peritoneal dialysis patients. The patients in this study all had end stage renal disease and were on renal replacement therapy. Other patients not on renal replacement were not assessed. In addition, patients in the other stages of chronic kidney disease were not studied. These groups are an area for future research.

\section{Conclusion}

This study assesses the factors impacting on the choice of renal replacement therapy. The generation of new data on these will have a profound impact both locally and regionally in decision making and management. With the increasing demands for renal replacement, these factors affecting modality of renal replacement can be used to better influence decision making among patients. Additionally, comorbidities contributing to the development of end stage renal disease can be addressed and preventative measures can be optimized.

\section{References}

[1] Liem, Y.S., Bosch, J.L. and Arends, L.R. (2007) Quality of Life Assessed with the Medical Outcomes Study Short-Form 36-Item Health Survey of Patients on Renal Replacement Therapy: A Systematic Review and Metaanalysis. Value in Health, 10, 390-397. http://doi.org/10.1111/j.1524-4733.2007.00193.x

[2] Cameron, J.I., Whiteside, C., Katz, J. and Devins, G.M. (2000) Differences in Quality of Life across Renal Replacement Therapies: A Meta-Analytic Comparison. American Journal of Kidney Disease, 35, 629-637.

[3] Makkar, V., Kumar, M., Mahajan, R. and Khaira, N.S. (2015) Comparison of Outcomes and Quality of Life between Hemodialysis and Peritoneal Dialysis Patients in Indian ESRD Population. Journal of Clinical and Diagnostic Research, 9, 28-31. http://doi.org/10.7860/JCDR/2015/11472.5709

[4] Mendes de Abreu, M., Walker, D.R., Sesso, R.C. and Ferraz, M.B. (2011) Health-Related Quality of Life of Patients Receiving Hemodialysis and Peritoneal Dialysis in São Paulo, Brazil: A Longitudinal Study. Value in Health, 13, 191-211. http://doi.org/10.3747/pdi.2011.00138

[5] García-Llana, H., Remor, E. and Sagas, R. (2013) Adherence to Treatment, Emotional State and Quality of Life in Patients with End-Stage Renal Disease Undergoing Dialysis. Psicothema, 25, 79-86. http://doi.org/10.7334/psicothema2012.96

[6] Bakewell, A.B., Higgins, R.M. and Edmunds, M.E. (2002) Quality of Life in Peritoneal Dialysis Patients: Decline over Time and Association with Clinical Outcomes. Kidney International Journal, 61, 239-248. http://doi.org/10.1046/j.1523-1755.2002.00096.x

[7] Khan, S., Bisnath, R., Jaipaul, J., Doodhai, J., Ramlal, D., et al. (2015) Screening for Chronic Kidney Disease in East Trinidad Using the National Kidney Foundation Guidelines. West Indian Medical Journal Supplement, 1-75. http://doi.org/10.19070/2572-7613-160007

[8] Fiebiger, W., Mitterbauer, C. and Oberbauer, R. (2004) Health-Related Quality of Life Outcomes after Kidney Transplantation. Health Related Quality of Life Outcomes, 2, 2. http://doi.org/10.1186/1477-7525-2-2

[9] Alvares, J., Cesar, C.C., Acurcio Fde, A., Andrade, E.I. and Cherchiglia, M.L. (2012) Quality of Life of Patients on Renal Replacement Therapy in Brazil: Comparison of 
Treatment Modalities. Quality of Life Research Journal, 21, 983-991. http://doi.org/10.1007/s11136-011-0013-6

[10] Laupacis, A., Keown, P., Pus, N., Krueger, H., Ferguson, B., Wong, C. and Muirhead, N. (1996) A Study of the Quality of Life and Cost Utility of Renal Transplantation. Kidney International Journal, 50, 235-242.

https://doi.org/10.1038/ki.1996.307

[11] Oliveira de Mendonça, A.E., Torres, G., Salvetti, M., Alchieri, J.C. and Fernandes Costa, I.K. (2014) Changes in Quality of Life after Kidney Transplantation and Related Factors. Acta Paulista de Enfermagem, 27, 287-292. http://doi.org/10.1590/1982-0194201400048

[12] Eduardo, N., Rodrigues, R. and Barata, C. (2015) Dyadic Relationship and Quality of Life of Patients with Chronic Kidney Disease. Brazilian Journal of Nephrology, 37, 315-322. http://doi.org/10.5935/0101-2800.20150051

[13] Liem, Y.S., Bosch, J.L. and Hunink, M.G. (2008) Preference Based Quality of Life of Patients on Renal Replacement Therapy: A Systematic Review and Meta-Analysis. Value in Health, 11, 733-741. http://doi.org/10.1111/j.1524-4733.2007.00308.x

[14] Panagopoulou, A., Hardalias, A., Berati, S. and Fourtounas, C. (2009) Psychosocial Issues and Quality of Life in Patients on Renal Replacement Therapy. Saudi Journal of Kidney Disease and Transplantation, 20, 212-218.

[15] Valderrabano, F., Jofre, R. and Lopez-Gomez, J.M. (2001) Quality of Life in End Stage Renal Disease Patients. American Journal of Kidney Disease, 38, 443-464. https://doi.org/10.1053/ajkd.2001.26824

[16] Sayin, A., Mutluay, R. and Sindel, S. (2007) Quality of Life in Haemodialysis, Peritoneal Dialysis and Transplantation Patients. Transplant Proceedings, 39, 3047-3053. https://doi.org/10.1016/j.transproceed.2007.09.030

[17] Soyibo, A.K. and Barton, E.N. (2007) Report from the Caribbean Renal Registry. West Indian Medical Journal, 56, 355.

[18] The National Organ Transplant Unit of Trinidad and Tobago (2016) Eric Williams Medical Sciences Complex, Mt Hope, Trinidad, West Indies.

[19] Roberts, L., Ramsaroop, K. and Seemungal, T. (2012) Survival Outcomes in Renal Transplantation in Trinidad and Tobago: SORTTT Study. West Indian Medical Journal, 61, 422-428. https://doi.org/10.7727/wimj.2012.201

[20] National Kidney Foundation (2002) KDOQI Clinical Practice Guidelines for Chronic Kidney Disease: Evaluation, Classification, and Stratification.

https://www.kidney.org/sites/default/files/docs/ckd_evaluation_classification_stratif ication.pdf

[21] International Standard Classification of Occupations of the United Nations. ISCO 08. http://www.ilo.org/public/english/bureau/stat/isco/

[22] World Health Organization (2008) Global Database on Body Mass Index: An Interactive Surveillance Tool for Monitoring Nutrition Transition.

http://apps.who.int/bmi/index.jsp

[23] World Health Organization (2012) International Classification of Diseases and Related Health Problems. ICD 10, 1-201. http://www.who.int/classifications/icd/ICD10Volume2_en_2010.pdf

[24] Tillin, T., Sattar, N., Godsland, I.F., Hughes, A.D., Chaturvedi, N., et al. (2015) Ethnicity Specific Obesity Cut-Points in the Development of Type 2 Diabetes Mellitus-A Prospective Study Including Three Ethnic Groups in the United Kingdom. Diabetic Medicine, 32, 226-234. http://doi.org/10.1111/dme.12576 
[25] Robinson, B.M., Akizawa, T., Jager, K.I., Kerr, P.G., Saran, R., et al. (2016) Factors Affecting Outcomes in Patients Reaching End Stage Kidney Disease Worldwide: Differences in Access to Renal Replacement Therapy, Modality Use, and Haemodialysis Practices. Lancet, 388, 294-306. http://doi.org/10.1016/S0140-6736(16)30448-2

[26] Gerasimoula, K., Lefkothea, L., Maria, L., Victoria, A., Paraskevc, T., et al. (2015) Quality of Life in Haemodialysis Patients. Materia Sociomedica, 27, 305-309. http://doi.org/10.5455/msm.2015.27.305-309

[27] Chen, M.C., Tsai, W.L., Tsai, I.C., Chan, S.W., Liao, W.C., et al. (2010) Arteriovenous Fistula and Graft Evaluation in Hemodialysis Patients Using MDCT: A Primer. American Journal of Roentgenology, 194, 838-847. http://doi.org/10.2214/AJR.09.3357

[28] Wasse, H., Kutner, N., Zhang, R. and Huang, Y. (2007) Association of Initial HD Vascular Access with Patient Reported Health Status and Quality of Life. Clinical Journal of the American Society of Nephrology, 2, 708-771. http://doi.org/10.2215/CJN.00170107

[29] Mohamed-Ali, V., Goodrick, S., Bulmer, K., Holly, J.M., Yudkin, J.S., et al. (1999) Production of Soluble Tumor Necrosis Factor Receptors by Human Subcutaneous Adipose Tissue In Vivo. American Journal of Physiology, 277, E971-E975.

[30] Demas, G.E., Drazen, D.L. and Nelson, R.J. (2003) Reductions in Total Body Fat Decrease Humoral Immunity. Proceedings of the Royal Society Biological Sciences, 270, 905-911. http://doi.org/10.1098/rspb.2003.2341

[31] Beddhu, S. (2004) The Body Mass Index Paradox and an Obesity, Inflammation, and Atherosclerosis Syndrome in Chronic Kidney Disease. Seminar in Dialysis Journal, 17, 229-232. http://doi.org/10.1111/j.0894-0959.2004.17311.x

[32] Kalantar-Zadeh, K., Abbott, K.C., Salahudeen, A.K., Kilpatrick, R.D. and Horwich, T.B. (2005) Survival Advantages of Obesity in Dialysis Patients. The American Journal of Clinical Nutrition, 81, 543-54. https://doi.org/10.1093/ajcn/81.3.543

[33] Ikizler, T.A. (2008) Resolved: Being fat is good for dialysis patients: The Godzilla Effect. Journal of the American Society of Nephrology, 19, 1059-1064. http://doi.org/10.1681/ASN.2007090983

[34] Fadem, S.Z., Walker, D.R., Abott, G., Friedman, A.L., Goldman, R., et al. (2011) Satisfaction with Renal Replacement Therapy and Education: The American Association of Kidney Patients Survey. Clinical Journal of the American Society of Nephrology, 6, 605-612. http://doi.org/10.2215/CJN.06970810

[35] Mehrotra, R., Marsh, D., Vonesh, E., Peters, V. and Nissenson, A. (2005) Patient Education and Access of ESRD Patients to Renal Replacement Therapies beyond In-Center Hemodialysis. Kidney International Journal, 68, 378-390. http://doi.org/10.1111/j.1523-1755.2005.00453.x

[36] United States Renal Data System (1997) The USRDS Dialysis Morbidity and Mortality Study: Wave 2. American Journal of Kidney Disease, 30, S67-S85. http://doi.org/10.1038/kisup.2015.2

[37] Finkelstein, F.O., Story, K., Firanek, C., Barre, P., Takano, T., et al. (2008) Perceived Knowledge among Patients Cared for by Nephrologists about Chronic Kidney Disease and End-Stage Renal Disease Therapies. Kidney International Journal, 74, 1178-1184. http://doi.org/.10.1038/ki.2008.376

[38] Morton, R.L., Tong, A., Howard, K., Snelling, P. and Webster, A.C. (2010) The Views of Patients and Careers in Treatment Decision Making for Chronic Kidney Disease: Systematic Review and Thematic Synthesis of Qualitative Studies. British 
Medical Journal, 340, c112. https://doi.org/10.1136/bmj.c112

[39] Givens, R. (2016) The United Network for Organ Sharing (UNOS).

http://optn.transplant.hrsa.gov/data/view-data-reports

[40] Rabbat, C.G., Thorpe, K.E., Russell, J.D. and Churchill, D.N. (2000) Comparison of Mortality Risk for Dialysis Patients and Cadaveric First Renal Transplant Recipients in Ontario, Canada. Clinical Journal of the American Society of Nephrology, 11, 5917-5922.

[41] Don, Y.K., Tammy, K.C., Myoung-Hee, K., Junhyug, N., Gunhee, K., et al. (2016) Superior Outcomes of Kidney Transplantation Compared with Dialysis: An Optimal Matched Analysis of a National Population-Based Cohort Study between 2005 and 2008 in Korea. Medicine, 95, e4352.

http://doi.org/10.1097/MD.0000000000004352 